ISSN 1823-626X

Malaysian Journal of Fundamental and Applied Sciences

available online at http://mjfas.ibnusina.utm.my

\title{
Comparison of EEG Source Localization using Meromorphic approximation to Fuzzy C-mean
}

\author{
Leila Saeidiasl $^{1 *}$, Tahir Ahmad ${ }^{1,2 *}$, Norma Alias ${ }^{1,2}$, Mehdi ghanbari $^{1}$ \\ ${ }^{1}$ Department of Mathematics Sciences, Faculty of Science, Universiti Teknologi Malaysia, 81310, UTM Johor Bahru, Johor Darul Ta'azim, Malaysia \\ ${ }^{2} I b n u$ Sina Institute for Fundamental Science Studies, University Technology Malaysia, 81310 Skudai, Johor, Malaysia \\ Received 2 January 2013, Revised 23 May 2013, Accepted 28 May 2013, Available online 4 June 2013
}

\begin{abstract}
Electroencephalography (EEG) is a neuroimaging technique for localizing active sources within the brain, from knowledge of electromagnetic measurements outside the head. Recognition of point sources from boundary measurements is an ill-posed inverse problem. InEEG, measurements are only accessible at electrode positions, the number of sources is not known a prior. This paper proposes a comparison between two approaches for EEG source localization. First method based on Meromorphic approximation techniques in the complex plane and second one belongs to EEG's method which is processed using Fuzzy C-Means (FCM). Comparison results on simulated data are used to verify the superior of the Meromorphic approximation with regarding to Fuzzy c-means, due to it provides the way for solving inverse problem of EEG source localization in $3 \mathrm{D}$ from boundary measurement based on Harmon function in the innermost layer .
\end{abstract}

| EEG source localization | Meromorphic Function| Fuzzy c-means |

(B) 2013 Ibnu Sina Institute. All rights reserved. http://dx.doi.org/10.11113/mjfas.v9n4.113

\section{INTRODUCTION}

EEG Source Localization techniques intends to localizing active sources inside the brain from measurements of the electromagnetic field they produce, which can be measured outside the head. This localization problem is commonly referred to as the inverse source problem of electroencephalography. They are ill-posed in general, mostly due to the lack of continuity and stability, but also to non-uniqueness $[1,2]$. By introducing reasonable a priori restrictions, the inverse problem can be solved and the most probable sources in the brain can be accurately localized [3]. One of the this assumption is about limitation of number of sources. When a limited number of sources are modelled as pointwise and dipolar, it has been verified that the inverse problem of source estimation has a unique solution [4].

Several families of techniques exist to solve the inverse source localization problem, when sources can be modelled as a small number of dipoles [5]. Dipole fitting methods must minimize a non-convex goal function, a result that is unstable with respect to the number of dipoles in the model [6]. When this number is supposed to be known in advance, an algebraic method has been offered in [4], which requires rank computation of related matrices.

In reality, one does not know this number a prior, and learning this model order is far from trivial [7]. The multiple signal classification (MUSIC) technique first utilizes a principal component analysis (PCA) to the measu- rements, and then recognizes a 'signal subspace' the analysis of which subsequently verifies the dipole positions [8]. Using the fact that MUSIC method can be applied if the sources are well modelled by a small number of dipoles. Beamforming is one of the most commonly used methods for estimating the active neural sources. The basic assumption in beamforming is that the sources are uncorrelated, which allows for estimating each source independent of the others [9].It can also estimate active sources, by scanning a region of interest, and by comparing the covariance of the measurement to that of the baseline, measured in time windows that do not contain the activity of interest [10].

This paper focuses on comparison between two approaches, which, like MUSIC and beamforming, requires no former information on the number of sources. However, unlike MUSIC or beamforming, which need as input successive measurements in a time window. Meromorphic approximation method belongs to a new category of source estimation algorithms that are based on harmonic analysis and best approximation theory, which is directly detect the sources as the singularities of the potential from boundary measurements[11].The second one is belong to fuzzy cmeans clustering algorithm, which has been widely applied to a vast variety of problem [12]. In the term of application, FCM has been altered and generalized in many ways in order to localize sources (cluster) and reduce the execution time of the algorithm for real -time classification purpose. Needless to point out that all the fuzzy clustering algorithms 
depend heavily on elements of Zadeh [19] fuzzy set theory and many of them are based entirely on the FCM algorithm, originally proposed by Dunn[13] and further extended by Bezdek [12]. The aim of FCM is to find cluster centre (sources) that minimize an objective function. It employs fuzzy partitioning such that a data point can belong to all groups with different membership grads between 0 and 1.In this paper, FCM clustering technique is applied to a large EEG data set from the epileptic patients to identify representative fuzzy membership values and cluster centre.

\section{METHODS}

\subsection{Source detection by Meromorphic approximation method function}

The head $\Omega=\cup_{\mathrm{i}=0}^{2} \Omega_{\mathrm{i}} \subset \mathbb{R}^{3}$ is modelled as a set of nested regions $\Omega_{\mathrm{i}} \subset \mathbb{R}^{3}, \mathrm{i}=0,1,2$ (brain, skull, scalp), separated by spherical interfaces $\mathrm{S}_{\mathrm{i}}$ (with $\mathrm{S}_{2}=\partial \Omega$ ), (figure 1) and with piecewise constant conductivityo, $\sigma_{\left.\right|_{\Omega_{\mathrm{i}}}}=\sigma_{\mathrm{i}}>0$.

Considering a macroscopic physical model of brain activity and using a quasi-static approximation of the Maxwell equations, the spatial behaviour of the electric potential $u$ in $\Omega$ is related to the distribution of $m$ dipolar sources located at $c_{\mathrm{k}} \in \Omega_{0} \quad$ (position)with moments $\mathrm{p}_{\mathrm{k}} \in \mathbb{R}^{3}$ by Poisson equation :

$$
\begin{cases}\nabla .(\sigma \nabla u)=\nabla \cdot \vec{J}^{p}=\sum_{k=1}^{m} p_{k} . \nabla \delta_{c_{k}} & \text { in } \Omega \subset R^{3} \\ \sigma \frac{\partial u}{\partial n}=\phi & \text { on } \quad \Gamma=\partial \Omega\end{cases}
$$

such that $g$ and $\phi$ denote the given potential and current flux on the scalp. The solution of the inverse EEG problem for recovering $\left(m, C_{k}, p_{k}\right)$ can be divided into two main steps:

\section{- Data transmission}

- Source Recovery

First category is data transmission from $S_{2}$ to $S_{0}$, which involves: Cortical Mapping and Harmonic function. Main application of cortical mapping is in EEG where potential measurements available at part of the scalp are used to reconstruct the potential and the current on the inner skull surface. More precisely, cortical mapping deals with the recovery of $u$ and $\partial_{\mathrm{n}} \mathrm{u}$ on the interfaces $\left(S_{1}, S_{2}\right)$, from a set of discrete measurements of $u$ on scalp electrodes. This problem is known as cortical mapping. Our Cortical Mapping solution uses the Symmetric Boundary Element (SBE) method, in which $u$ is discretized with piecewise linear polynomials on each surface of the head model, and $\sigma \frac{\partial u}{\partial n}$ is discretized with constant polynomials on each surface of the head model. This method originally presented in [14].

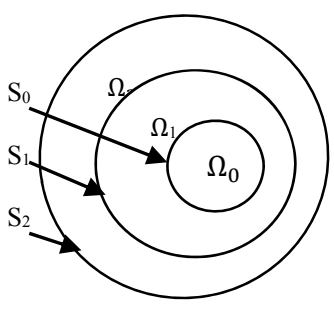

Fig. 1 The head is modelled as three nested volumes $\Omega_{i}$, with the spherical interfaces $S_{i}$.

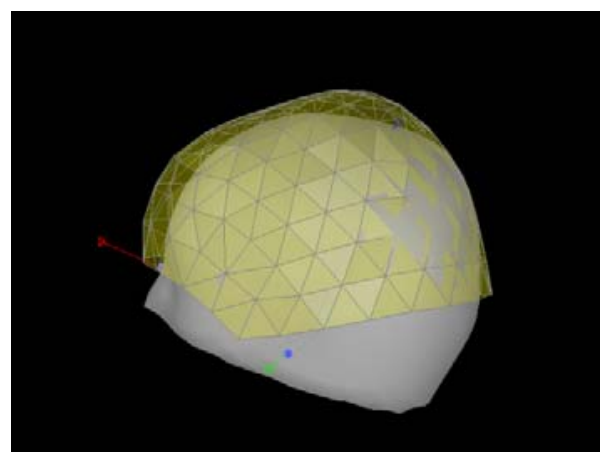

Fig. 2 Generating Boundary element meshes on the scalp [21].

The potential $u$ inside the head satisfies the Poisson equation $\nabla \cdot(\sigma \vec{\nabla} u)=\nabla \cdot \vec{J}^{p} \quad$ where $\nabla \cdot \vec{J}^{p} \quad$ represents the primary sources localized inside the brain $\Omega_{0}$. Data is the pointwise values of $u$ measured by electrodes on the scalp $S_{2}$. Parameters $u$ and $\partial_{\mathrm{n}} \mathrm{u}$ on $S_{0}$, were found in this step. Figure 3 represents the cortical step from outer layer to innermost layer (brain).

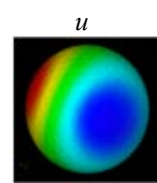

Scalp

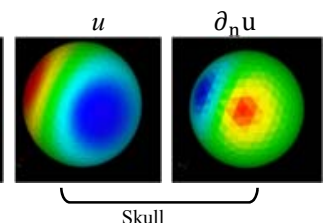

Skull

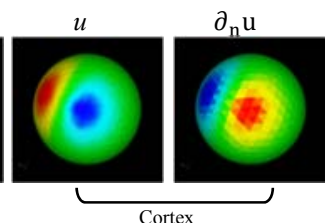

Cortex
Fig. 3 Cortical Mapping step (data transmission) from electrode data to the cortical surface [11].

cortical mapping has supplied the potential $u=g$ and its normal derivatives $\frac{\partial u}{\partial n}=\varphi$ on the surface of cortex $S_{0}$. The potential $u$ satisfies in $\Omega_{0}$ an equation of the form:

$$
\begin{cases}\Delta u=f=\sum_{k=1}^{N} p_{k} \cdot \nabla \delta_{c_{k}} & \text { in } \Omega_{0} \\ \frac{\partial u}{\partial n}=\varphi \quad, u=g & \text { on } S_{0}\end{cases}
$$

Next step is finding (again up to the addition of a harmonic function) the potential $u$ created by and the dipolar sources 
$\left(\mathrm{C}_{\mathrm{k}}, \mathrm{p}_{\mathrm{k}}\right)$ located inside $\Omega_{0}$, which is a solution to Eq. (2).Firstly, one try to find the part $u_{a}$ of the potential $\mathrm{u}$ that harmonic outside the ball $\Omega_{0}$, and contain all the information on the distribution of sources on $\mathrm{S}_{0}$.i.e., filtering out possible outer sources with spherical harmonics by keeping only the information related to the effective inner sources in $\Omega_{0}$ provides a function [15].

$$
\mathrm{u}_{\mathrm{a}=\sum_{\mathrm{k}=1}} \frac{\left\langle\mathrm{p}_{\mathrm{k}}, \mathrm{x}-\mathrm{C}_{\mathrm{k}}\right\rangle}{4 \pi\left\|\mathrm{x}-\mathrm{C}_{\mathrm{k}}\right\|^{3}} \quad, \mathrm{x} \neq \mathrm{C}_{\mathrm{k}}
$$

Where the brackets \langle\rangle indicate the scalar product in $\mathbb{R}^{3} \times$ $\mathbb{R}^{3}$. Reminder that, $\mathrm{u}_{\mathrm{a}}$ include all information about distribution of sources on $S_{0}$ and also,

$$
\Delta \mathrm{u}_{\mathrm{a}}=\Delta \mathrm{u}=\mathrm{f} \quad \text { in } \Omega_{0}
$$

But, $\Delta \mathrm{u}_{\mathrm{a}}=0$ in $\mathbb{R}^{3} \backslash \Omega_{0}$ since all sources are inside $\Omega_{0}$. From available data on $S_{0}$, the solution $u$ to equation (3.17) in $\Omega_{0}$, is assumes the form:

$$
\mathrm{u}(\mathrm{x})=\mathrm{h}(\mathrm{x})+\mathrm{u}_{\mathrm{a}}(\mathrm{x})=\mathrm{h}(\mathrm{x})+
$$$$
\sum_{\mathrm{k}=1}^{\mathrm{n}} \frac{\left\langle\mathrm{p}_{\mathrm{k}}, \mathrm{x}-\mathrm{C}_{\mathrm{k}}\right\rangle}{4 \pi\left\|\mathrm{x}-\mathrm{C}_{\mathrm{k}}\right\|^{3}} \quad, \mathrm{x} \in \Omega_{0} \backslash\left\{\mathrm{C}_{\mathrm{k}}\right\}
$$

where,$h$ harmonic function in $\Omega_{0}$. Ultimately, to recover the Ck inside $\Omega_{0}$, the knowledge of the singular function $u_{a}$ is required on $S_{0}$. This can be deduced from available boundary data by expanding $\mathrm{u}$ on bases of spherical harmonics [16].

The second category in the resolution of the inverse problem (IP) is the best rational approximation on planar sections (Source Recovery):

pointwise values of $\mathrm{u}_{\mathrm{a}}$

Localisation of sources $\mathrm{C}_{\mathrm{k}}$

$2 D$ Meromorphic approximation on planar sections of the boundary

- $\quad$ Slice $\Omega_{0}$ along a family of planes $\Pi_{\mathrm{p}}: \Pi_{\mathrm{p}} \cap \mathrm{s}_{0}=\Gamma_{\mathrm{p}}$ (circles).

- From pointwise values of the singular part $u_{a}$ on $\Gamma_{\mathrm{p}}$, approximate $f_{p}=\left(u_{a}\right)^{2}$ the square of harmonic function on $\Gamma_{\mathrm{p}}[15,16]$.

- $\mathrm{f}_{\mathrm{p}}$ is a meromorphic function, analytic outside $\mathrm{D}_{\mathrm{p}}=\Pi_{\mathrm{p}} \cap \mathrm{s}_{0}$ with singularities $\xi_{\mathrm{k}, \mathrm{p}}$ inside $D_{p}$ which are strongly linked with the sources $C_{k}$.

- Approximate the $\xi_{\mathrm{k}, \mathrm{p}}$ by $\widetilde{\xi_{\mathrm{k}, \mathrm{p}}}$ the poles of the best $\mathrm{L}^{2}$ or $\mathrm{L}^{\infty}$ rational approximation to $f_{p}$ on $\Gamma_{\mathrm{p}}$ (degree $\geq m$ for a sphere), the poles $\widetilde{\xi_{\mathrm{k}, \mathrm{p}}}$ accumulate to the singularities $\xi_{\mathrm{k}, \mathrm{p}}$ [16]

- Varying $p$, this allows us to approximately locate the $m$ sources $C_{k}$ in $\Omega_{0}$.

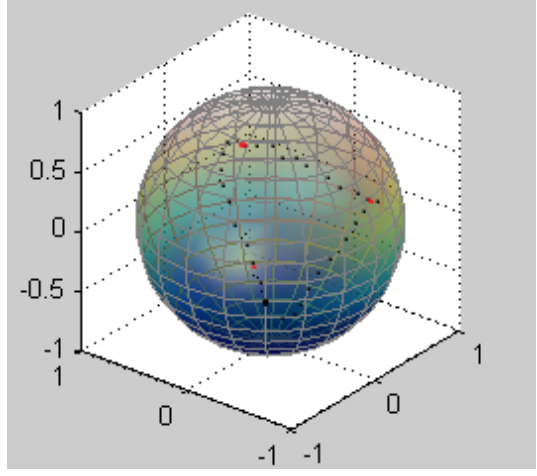

Fig. 4 Estimated sources positions (red) for different slicing directions in 3D.

\subsection{Source detection by Fuzzy c-Means}

Fuzzy clustering is used in grouping the potential difference of EEG signals during seizure attack. This clustering is important in identifying the number of cluster differences during the different stages of seizure attack. The type of clustering that is used in this paper is Fuzzy $c$ Means (FCM) clustering.

FCM algorithm is an iterative partitioning method that produces optimal $c$-partitions. The method compute the cluster centre (sources) and generates the class membership matrix.

The first step of FCM algorithm is to generate an initial random membership matrix and use this random membership matrix as the weight of each sample that belongs to each cluster, and then compute the centroid of each cluster. The new cluster centres are used to update the membership matrix. The update membership matrix is compared to the previous ones. If the difference is greater than some threshold, then iteration is computed, otherwise the algorithm is stopped.

FCM algorithm can be presented in four primary steps as follows:

Step 1: Randomly initialize the membership matrix $(U)$ that has constraints:

$\sum_{\mathrm{i}=1}^{\mathrm{c}} \mathrm{u}_{\mathrm{ij}}=1 \quad \forall \mathrm{j}=1, \ldots, \mathrm{n}$

Step2: Calculate centroids $\left(v_{i}\right)$ by using

$$
\mathrm{v}_{\mathrm{i}}=\frac{\sum_{\mathrm{j}=\mathrm{i}}^{\mathrm{n}} \mathrm{u}_{\mathrm{ij}}^{\mathrm{m}} \mathrm{x}_{\mathrm{j}}}{\sum_{\mathrm{j}=1}^{\mathrm{n}} \mathrm{u}_{\mathrm{ij}}^{\mathrm{m}}}
$$

Step3: The objective of the FCM algorithm is to minimize the FCM cost function formulated below. Stop if its improvement over previous iteration is below a threshold. 


$$
\mathrm{J}_{\mathrm{m}}=\mathrm{J}\left(\mathrm{U}, \mathrm{v}_{1}, \mathrm{v}_{2}, \ldots, \mathrm{v}_{\mathrm{n}}\right)=\sum_{\mathrm{i}=1}^{\mathrm{c}} \mathrm{J}_{\mathrm{i}}=\sum_{\mathrm{i}=1}^{\mathrm{c}} \sum_{\mathrm{j}=1}^{\mathrm{n}} \mathrm{u}_{\mathrm{ij}}^{\mathrm{m}} \mathrm{d}_{\mathrm{ij}}^{2}
$$

Step4: Compute a new $U$ using equation below then go to step 2.

$$
\mathrm{u}_{\mathrm{ij}}=\frac{1}{\sum_{\mathrm{k}=1}^{\mathrm{c}} \frac{\mathrm{d}_{\mathrm{ij}} \frac{2}{\mathrm{~d}} \mathrm{~d}_{\mathrm{kj}}}{\mathrm{m}-1}}
$$

$\mathrm{u}_{\mathrm{ij}}$ is between 0 and 1 ;

$\mathrm{v}_{\mathrm{i}}$ is the centres of cluster $i$;

$\mathrm{d}_{\mathrm{ij}}$ is the Euclidian distance between $i$ th centre $\left(v_{i}\right)$ and $j$ th data point where $d_{i j}^{2}=\left\|x_{j}-v_{i}\right\|^{2}$;

$\mathrm{m} \in[1, \infty)$ is a weighting exponent [21].

\subsubsection{Cluster validity}

Cluster validity is a difficult problem that is crucial for the practical application of fuzzy clustering techniques. In addition note that, there are many purposes to employ cluster validity measures [12]. One of the most important applications is to estimate the number of clusters. Partition coefficient is one of the cluster validity measures that it is described as following:

$$
\mathrm{F}(\mathrm{U} ; \mathrm{c})=\frac{1}{N} \sum_{i=1}^{c} \sum_{k=1}^{N}\left(u_{k i}\right)^{2}
$$

Another type of cluster validity measure is Partition entropy:

$$
E(U ; C)=-\frac{1}{N} \sum_{i=1}^{c} \sum_{k=1}^{N} u_{k i} \log u_{k i}
$$

The clusters were judged better when one of these measures is larger.

\section{RESULTS \& DISCUSSION}

Numerical results from first method obtained with FindSources3D, a Matlab code that implements the above algorithm [17]. Two datasets were simulated with OpenMEEG [23], which implements the symmetric boundary element method [18]. Data at the scalp level was considered, potential measured by 128 electrodes, and also at the cortex level with 642 points mesh, see figure 2, which is extract from Brainstorm software [22], in order to test the influence of the cortical mapping step on the quality of the source estimation. The spherical three-layer is considered for head model. After the cortical mapping step, the part $u_{a}$ of the potential $u$ was found, which is harmonic outside the cortex and still contains on $\mathrm{S}_{0}$ all the information on the distribution of sources. Furthermore, using harmonic function $u_{a}$ (Meromorphic approximation), this step deals with the computation of the singularities $s_{k, p}$ from the sliced boundary data. The localization of $s_{k, p}$, which then leads to the $C_{k}$ (sources), see Figure 4.

For the second method, MATLAB software was considered to obtain the fuzzy membership values and its cluster centre. Initially, choosing the value of exponent for the partition matrix was needed, which is $m=2$; maximum number of iteration is 100 and the minimum of improvement is $1 \times 10^{-5}$.Then, follow the 4 steps as described in section 2.2 to determine fuzzy membership values, and its cluster centre for same real data. Figure 5 shows the number of cluster centre which are more signal activities occur from $t=4$ until $t=7$ and start to die out at $t$ $=8$. This result can lead to a founding of where a patient is prone to have seizure attack. Although, the fuzzy $c$-means algorithm partitions a data set into a predefined three number of clusters, whether or not the data set actually contains three clusters .For this reason, cluster validity measure is used to find the optimal number of cluster for every second. Following table, present the optimal number of cluster centre.

Table 2 Three cluster centre with its potential Differences

\begin{tabular}{|l|c|c|c|l|}
\hline Cluster centre & $\mathrm{X}$ & $\mathrm{Y}$ & $\mathrm{Z}$ & $\begin{array}{l}\text { Potential } \\
\text { difference }\end{array}$ \\
\hline $\mathrm{V}_{1}$ & -0.7036 & -0.3095 & -0.19088 & 3.7643 \\
\hline $\mathrm{V}_{2}$ & 1.0023 & 0.5789 & 0.5480 & 22.3468 \\
\hline \hline $\mathrm{V}_{3}$ & 2.2345 & 1.1453 & 1.5432 & 13.3790 \\
\hline
\end{tabular}

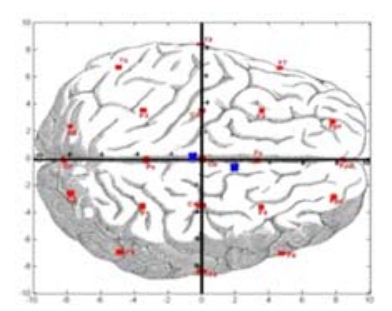

$\mathrm{t}=1$

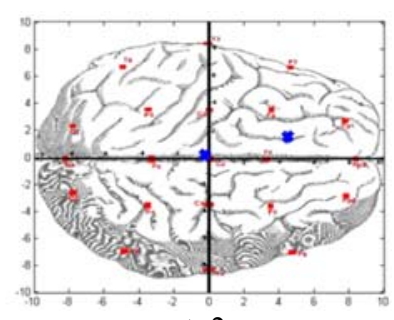

$\mathrm{t}=2$

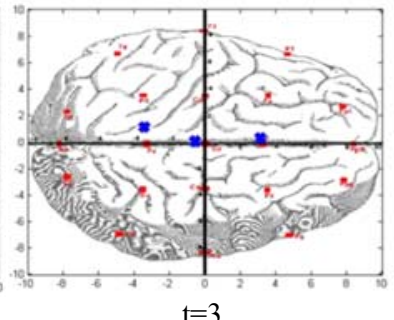

$t=3$

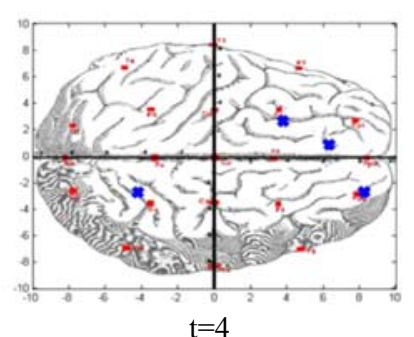

$\mathrm{t}=4$ 

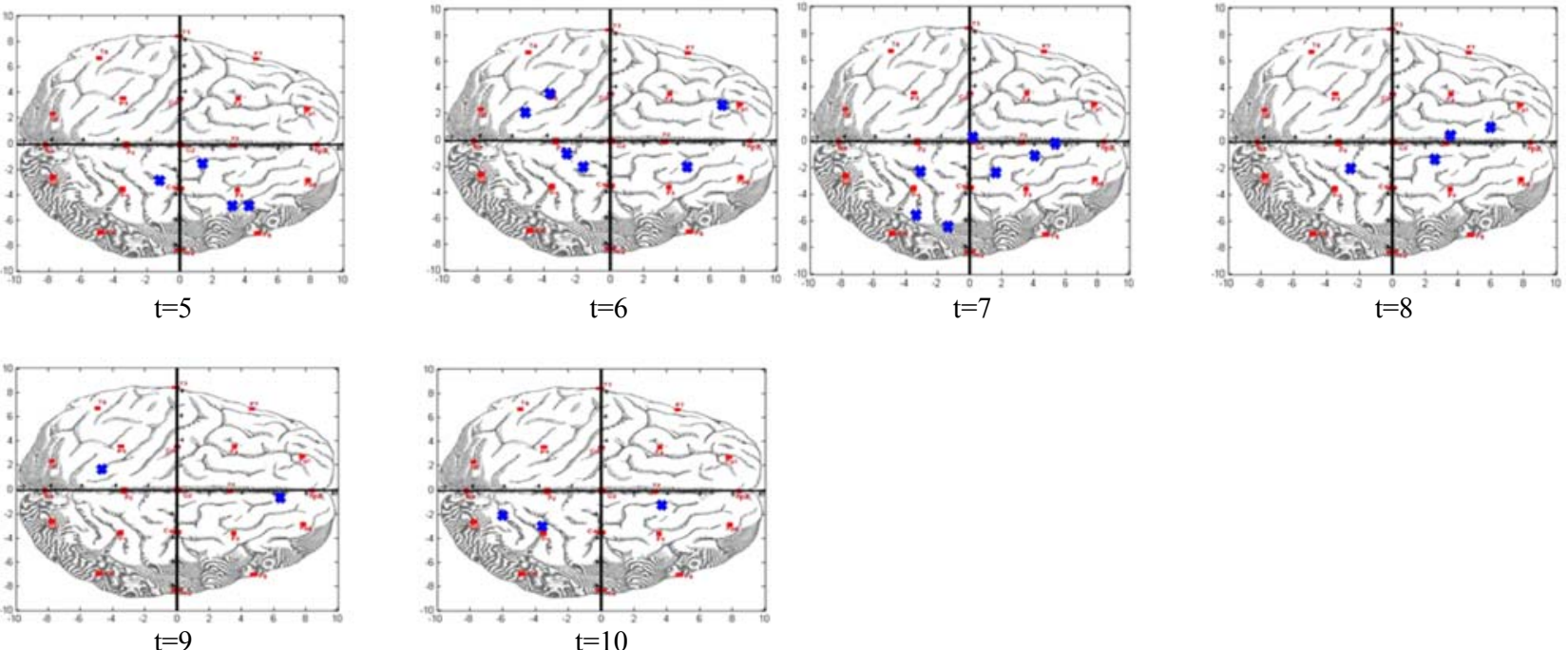

Fig. 5 position of cluster centre for duration of 10 seconds. Locations of sensors on the surface of the patient's head are in red, the cluster's centres for signal are in blue.

\section{CONCLUSION}

Some insights concerning the resolution of a source estimation problem were presented. The techniques rely on constructive approximation. They are robust and efficient toward the EEG inverse source problem, as illustrated by preliminary numerical result. Fuzzy c-means enables the possibility of clustering the EEG signals while the patient is having a seizure attack. The center of the cluster (sources) has been identified when the attack occurred.Result shows most signal activities occur from $t=4$ until $t=7$ and start to die out at $t=8$.

Meromorphic approximation technique shows better result than Fuzzy c-means clustering. Since it can directly localize recovering an unknown number of dipole sources within a homogeneous domain (not a nested geometry) from measurements of the potential and its normal derivative on the boundary. The superiority of the Meromorphic approximation compare to Fuzzy c-means is that source estimation accomplish after data transmission at the cortical level with harmonic function. On the other hand, the fuzzy C-mean relied on electrodes positions on the scalp. Another limitation of Fuzzy c-means method is that, it is time dependent. This fact degrades the clustering accuracy due to large noises in some time slices, hence reduce the localization accuracy.

\section{ACKNOWLEDGEMENT}

The authors would like to thank to Universiti Teknologi Malaysia (UTM) and Ministry of Higher Education (MOHE) for granting the Research University Grant (GUP) for this research. The grant received is under the Vote No. Q.J13000.7126.02H36.

\section{REFERENCES}

[1] S .Vessella, Inverse Problems, 8.6 (1992) 911-9117.

[2] V .Isakov, Inverse Problems for Partial Differential Equations, Springer, USA, 1998.

[3] R. D. Pascual-Marqui, ISBET Newslett, 6 (1995) 16-28.

[4] A.El Badia, and T.Ha-Duong, Inverse Problems, 16 (2000) 651663

[5] M .Scherg, T. Bast, and P .Berg, J. Clin. Neurophysiol. 16 (1999) 214-224.

[6] B. Cuffin, IEEE Trans. Biomed. Eng., 42 (1995) 68-71.

[7] C.G.B'enar, R. N.Gunn, C.Grova, B.Champagne , and J.Gotman ,IEEE Trans. Biomed. Eng., 52 (2005) 401-413.

[8] J .C .Mosher, P. S .Lewis, and R .M. Leahy, IEEE Trans. Biomed. Eng., 39 (1992) 541-553.

[9] A.Bahramisharif, M. A.J. Gerven, J.M. Schoffelen, Z.Ghahramani, and T. Heskes, Machine Learning and Interpretation in Neuroimaging. Springer Berlin Heidelberg, 2012, 148-155.

[10] B. D. Van Veen, and K .M .Buckley, IEEE Acoust. Speech Signal Process Mag., 5 (1988) 4-24.

[11] M. Clerc, J .Leblond, J. P. M,armorat, and T. Papadopoulo, Inverse Problems, 28 (2012) 1-24.

[12] J.C .Bezdek, Pattern Recognition with Fuzzy Objective Function Algorithms. Plenum Press, New York, 1981.

[13] J.C .Dunn, and J.Cybern, 3 (1974) 32-57.

[14] M .Clerc, and J .Kybic., Inverse Problems 23 (2007) 589-601.

[15] L .Baratchart, J .Leblond, and J.P. Marmorat, Electron. Trans. Numer. Anal., 25(2006) 41-53.

[16] J .Leblond, C .Paduret, S .Rigat and M. Zghal, Inverse Problems 24 (2008) 1-38.

[17] R .Bassila, M .Clerc, J .Leblond, J.P. Marmorat and T. Papadopoulo, FindSources3D software, http://wwwsop.inria.fr/apics/FindSources3D, (2008)

[18] J .Kybic, M .Clerc, T .Abboud, O .Faugeras, R .Keriven and T. Papadopoulo, IEEE Trans. Med. Imaging, 24 (1965)12-28.

[19] L.A. Zadeh, 8 (1965) 338-353.

[20] F .Zakaria, PhD Thesis, Universiti Teknologi Malaysia, 2008.

[21] F .Tadel, S .Baillet, J.C .Mosher, D .Pantazis, R.M. Leahy, Computational intelligence and neuroscience, 2011 (2011) $1-13$.

[22] A. Gramfort, T. Papadopoulo, E. Olivi, and M. Clerc. Biomedical Engineering Online 9 (2010) 1-20. 